\title{
Metallographic Observations of Delayed Hydride Cracking in $\mathrm{Zr}-2.5 \mathrm{Nb}$
}

\author{
Z. Pan and A. Lockley
}

Materials and Mechanics Branch, Chalk River Laboratories, Atomic Energy of Canada Ltd. Chalk River, Ontario, CANADA, K0J 1J0

$\mathrm{Zr}-2.5 \mathrm{Nb}$ is an alloy used to make pressure tubes in CANDU reactors for its combination of corrosion resistance, good mechanical properties, and low neutron capture cross-section. Studies are undertaken of life-limiting phenomena for $\mathrm{Zr}-2.5 \mathrm{Nb}$ to ensure that these phenomena are well understood and that pressure tubes are not operated in a compromised state. One such phenomenon is Delayed Hydride Cracking (DHC) [1,2]. DHC is a time-dependent cracking mechanism based on diffusion of hydrogen in the presence of a tensile stress to the tip of a flaw, followed by nucleation, growth and fracture of a brittle hydride phase along appropriately oriented crystal planes. Repetition of these processes, aided by a thermal cycle, can lead to crack propagation in zirconium alloys. DHC can be prevented by ensuring there are no good crack initiation sites, that the hydrogen concentration is not sufficient to precipitate hydrides, or that crystal planes susceptible to cracking are not under high tensile stress. In the present work, eight simulated flaws were machined into the inside surface of a section of pressure tube containing 38 $\mu \mathrm{g} / \mathrm{g}$ of hydrogen. The tube assembly was thermally cycled, with an internal pressure higher than typically experienced by pressure tubes to initiate DHC. Optical metallography was used to examine the region under the simulated flaws tip. The purpose of this work is to characterize a DHC fracture from a simulated flaw by metallography. The typical DHC morphology observed is shown in Figure 1, and is consistent with earlier observations [1,2]. The key features of the observed DHC are its flat surface on the radial-longitudinal plane of the pressure tube due to hoop stress and crystallographic texture in the pressure tube, and its semi-elliptical shape due to different crack growth velocity in radial and axial directions. The coloured regions are the consequence of different oxide thickness on distinct stages of crack growth.

The evidence of the DHC initiation from hydrides at the flaw tip is found on the cross section of a simulated flaw, where the metallographic observation of hydride distribution around the flaw tip area indicates the presence of re-orientated, longer and denser hydride precipitates, Figure 2. The cross section surface was etched using a standard etching technique to expose the hydrides. Quantitative image analysis was used to determine the hydride reorientation degree and average length per unit area as a function of position on the cross sections. It was observed that both reorientation degree and length of hydride are the highest at the flaw tip while they are the lowest in the region remote from the flaw. Along the axial direction of the flaw, the hydride length and reorientation degree in the first cross section, close to the location of high residual stress, are apparently higher than that in other two cross sections far from this location. Measurements of hydrogen concentration, residual stress and mechanical properties in the failed tube section were conducted. It is shown that the observed hydride morphology and the DHC initiation in the pressure tube section during thermal cycling are dependent on the total hoop stress, hydrogen concentration and thermal cycling pattern.

References:

[1] R. Dutton, Canadian Metall. Quarterly, Vol.17, No. 1-4 (1978), pp.16-25.

[2] B.A. Cheadle el al, "Zr in the Nuclear Industry", ASTM STP 939, (1987) pp.224-239. 


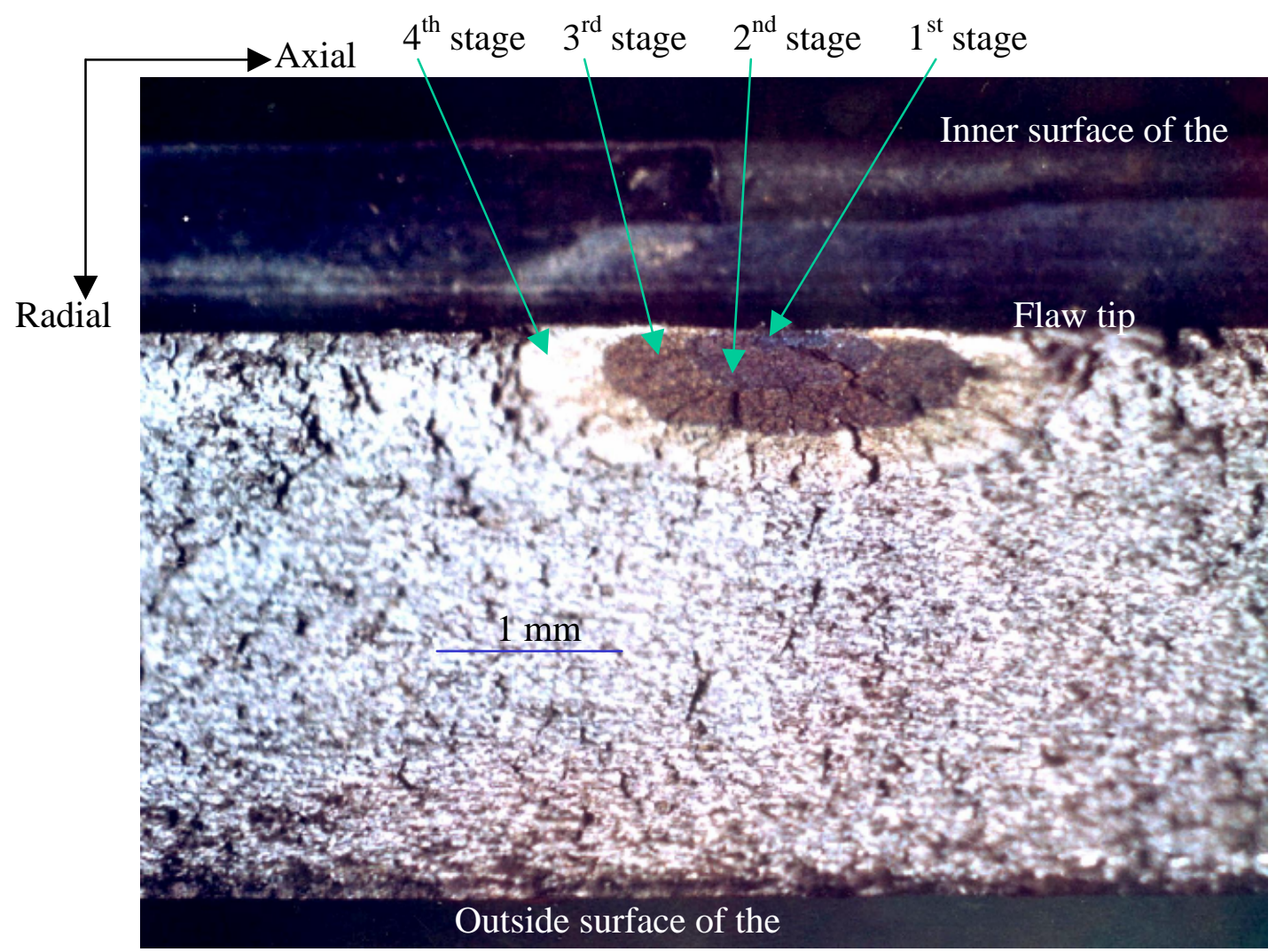

Figure 1 A typical DHC morphology on the broken-open surface. Four growth stages of the DHC from fifth to eighth thermal cycles can be clearly distinguished.

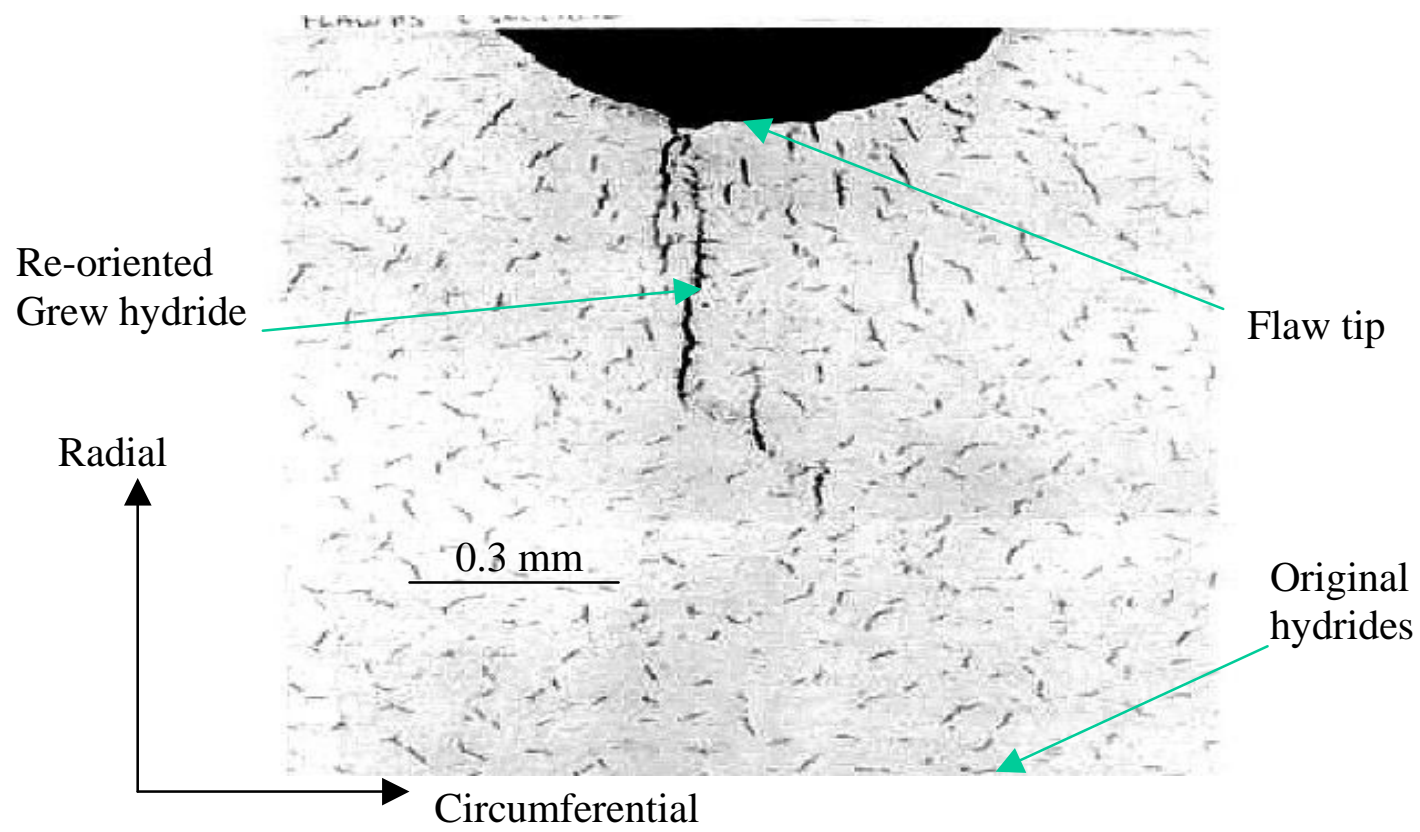

Figure 2 Hydride reorientation and growth under the flaw tip on a cross section, located at the position of high residual stress and high concentration of hydrogen. 